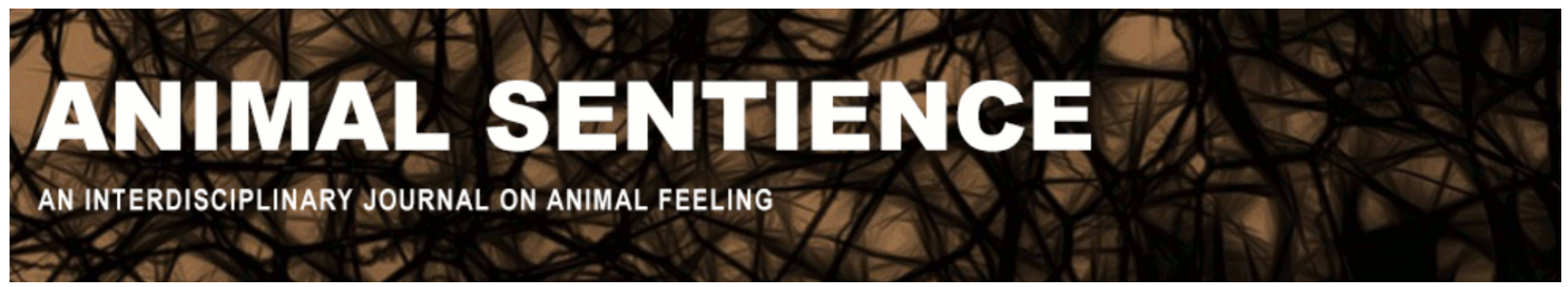

Found, Rob (2017) Individual variation in fear behaviour. Animal Sentience 15(3) DOI: $10.51291 / 2377-7478.1273$

Date of submission: 2017-12-09

Date of acceptance: 2017-12-12

(c) (i)




\title{
Individual variation in fear behaviour
}

Commentary on Beauchamp on Fear \& Vigilance

\author{
Rob Found \\ University of Alberta
}

\begin{abstract}
Individual variation in behaviour adds another layer of complexity to studies of animal responses to fear and risk, but when individual responses are consistent, and correlated with other behaviours, these patterns can provide a structure that furthers our understanding. I interpret Beauchamp's review of vigilance and fear from the perspective of my own studies of elk personality. Wild elk responses to changing stimuli (benign or aversive) illustrate how personality is a key factor in explaining variation in vigilance and fear behavior. Personality even influences how wildlife can learn to assess risk and adjust their fear responses accordingly.
\end{abstract}

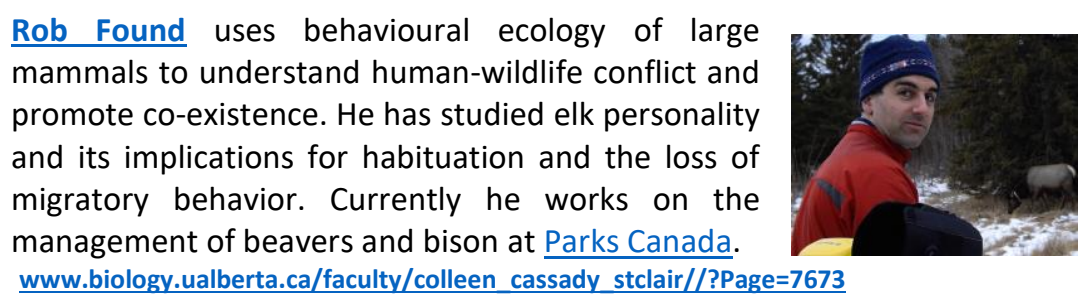

In his review of vigilance and fear, Beauchamp (2017) boldly tackles this fascinating imbroglio of animal behaviour by outlining the various nuances, mitigating factors, taxonomic variations, and seeming inconsistencies in measuring vigilance, and feelings and responses to fear in general. I suggest that more attention should be devoted to the implications of consistent individual variation in behavior - what animal behaviourists call coping styles (Koolhaas et al., 2007), temperament (Reale et al., 2007), behavioural syndromes (Sih et al., 2004) or more commonly, personality.

It is certainly easier to see patterns in behaviour if we assume that all animals behave in the same way - given the same stimuli, location, and context - so one might think that attributing variation in vigilance to personality would muddy the issue even further. Instead, I think that personality can provide a systematic structure for interpreting responses to fear. When individuals in a group appear to be exhibiting vigilance in ways, times, and locations that obscure observable patterns, ordering or categorizing individuals according to their personalities can reveal patterns in fear and vigilance.

I conducted one of the first comprehensive studies of personality in a wild ungulate, quantifying 6 different personality traits in wild elk (Cervus canadensis) in the Canadian Rocky Mountains (Found \& St. Clair, 2016). Using multivariate techniques, we used these six correlated behaviours to define a bold-shy gradient of personality in elk. Bold elk contrasted with shy elk in having shorter flight response distances (FRD), adopting peripheral rather than central positions within the herd, being less responsive to novel sound stimuli, exhibiting more neophilia towards novel objects, socially dominating shy elk, and devoting less time to vigilance. 
A re-examination of our raw data (Found \& St. Clair, 2016) provides an example of the risk of drawing conclusions about behavior when observations are made on unmarked, "generic" animals. When I compared the proportion of time elk spent on vigilance, to whether that elk was in an exterior or interior position within the herd, there was no correlation whatsoever. When I looked at the mean vigilance and positioning of each known individual elk (marked with ear tags), I found vigilance and internal positioning were strongly correlated $(r=$ 0.94). The pattern linking these two responses to risk only emerges only when we look at it through the lens of personality.

Beauchamp lists flight response distance (FRD) as another measure of fear that should be correlated with vigilance, if vigilance is in fact a measure of fear. When we looked at individually consistent vigilance and FRD behavior, such a correlation did in fact emerge in elk ( $r$ $=0.53$; Found \& St. Clair, 2016). Combining these results with Beauchamp's hypothesis thus leads to the conclusion - which should not surprise us given what we know about our own human personalities - that sensations and responses to fear are key components of the personalities of wild animals.

Beauchamp notes that vigilance behavior is "costly to perform," but a flight response, because it requires physical relocation, is perhaps even more costly to perform. We tested whether there was individual variation in how elk assessed and responded to risk (i.e., felt fear), by approaching elk benignly, at a regular walking speed, and measuring their FRD. We then repeated this to see whether individual responses would change as a result of experiencing these consecutive benign approaches. Bold elk successively decreased their FRD, whereas shytype elk did not (Found \& St. Clair, 2017). This shows the importance of personality in habituation, which, in the context of our discussion, is essentially the process of losing fear and/or responsiveness to fear (Rankin et al., 2009).

Were bold elk desensitizing to fearful stimuli and actually feeling less fear than shy elk, or were bold elk better at assessing risk and learning how to respond to fearful stimuli more appropriately? Can we separate these two processes? As Beauchamp notes, it can be hard to quantify the feeling of fear in wild animals. However, if certainly personality types are better at learning to gauge and respond to changing risk, the when elk encounters with humans are stressful and aversive, bold elk should increase their flight response distances more than shy elk. In a second experiment we did loud, highly stressful predator-like aversive chases at running speed, 10 minutes long (Found \& St. Clair, 2017). When encounters with humans were aversive, bold elk increased their FRD to the same levels as shy elk (which means the magnitude of those increases were much higher in bold elk). Three months later, when we stopped the aversive conditioning, the same bold elk once again began exhibiting very low flight response distances, whereas shy elk responses remained high. Shy elk were behaving sub-optimally, by having costly fear responses even when they didn't need to, while bold elk exhibited their fear responses only when it was actually beneficial. Since we had found that the flight response was correlated with vigilance in the elk we studied, I suspect that if we had used vigilance as our dependent variable, we would have seen similar personality-dependent flexibility in response to fear.

In his target article, Beauchamp has gathered in one place the multitude of factors influencing vigilance and fear in general. As ecologists move forward, we need to better unite this field of animal behaviour with the growing body of research exploring the implications of animal personality. My own research on elk has shown that rather than being just noisy variance 
complicating our understanding of fear and responses to risk, when individual variation is consistent, and correlated with other personality traits, it can provide a structure that provides greater insights into vigilance behaviour.

\section{References}

Beauchamp, G. 2017. What can vigilance tell us about fear? Animal Sentience 15(1).

Found, R. \& St. Clair, C.C. 2016. Behavioural syndromes predict loss of migration in wild elk. Animal Behaviour 115: 35-46.

Found, R. \& St. Clair, C.C. 2017. Ambidextrous ungulates have more flexible behavior, bold personalities and migrate less. Royal Society Open Science 4: 160958.

DOI: $10.1098 /$ rsos.160958

Koolhaas, J.M., deBoer, S.F., Buwalda, B. \& van Reenan, K. 2007. Individual variation in coping with stress: A multidimensional approach of ultimate and proximate mechanisms. Brain, Behaviour and Evolution 70: 218-226.

Rankin, R.A., Abrams, T., Barry, R.J., Bhatnagar, S., Clayton, D.F., Colombo, J., Coppola, G., Geyer, M. A., Glanzman, D.L., Marsland, S., McSweeney, F.K., Wilson, D.A., Wu, C-F. \& Thompson, R.F. 2009. Habituation revisited: An update and revised description of the behavioral characteristics of habituation. Neurobiology of Learning and Memory 92: 135-138.

Reale, D., Reader, S.M., Sol, D., McDougal, P.T. \& Dingemanse, J. 2007. Integrating animal temperament within ecology and evolution. Biological Review 82: 291-318.

Sih, A., Bell, A. \& Johnson, J.C. 2004. Behavioural syndromes: An ecological and evolutionary overview. Trends in Ecology and Evolution 19: 372-378. 